Swarthmore College

Works

$12-20-2011$

\title{
A Search For The Transit Of HD 168443b: Improved Orbital Parameters And Photometry
}

G. Pilyavsky

S. Mahadevan

S. R. Kane

A. W. Howard

D. R. Ciardi

See next page for additional authors

Follow this and additional works at: https://works.swarthmore.edu/fac-physics

Part of the Astrophysics and Astronomy Commons

Let us know how access to these works benefits you

\section{Recommended Citation}

G. Pilyavsky, S. Mahadevan, S. R. Kane, A. W. Howard, D. R. Ciardi, C. De Pree, D. Dragomir, D. A. Fischer, G. W. Henry, Eric L.N. Jensen, G. Laughlin, H. Marlowe, M. Rabus, K. Von Braun, J. T. Wright, and X. X. Wang. (2011). "A Search For The Transit Of HD 168443b: Improved Orbital Parameters And Photometry". Astrophysical Journal. Volume 743, Issue 2. DOI: 10.1088/0004-637X/743/2/162 https://works.swarthmore.edu/fac-physics/48

This work is brought to you for free by Swarthmore College Libraries' Works. It has been accepted for inclusion in Physics \& Astronomy Faculty Works by an authorized administrator of Works. For more information, please contact myworks@swarthmore.edu. 


\section{Authors}

G. Pilyavsky, S. Mahadevan, S. R. Kane, A. W. Howard, D. R. Ciardi, C. De Pree, D. Dragomir, D. A. Fischer, G. W. Henry, Eric L.N. Jensen, G. Laughlin, H. Marlowe, M. Rabus, K. Von Braun, J. T. Wright, and X. X. Wang 


\title{
A SEARCH FOR THE TRANSIT OF HD 168443b: IMPROVED ORBITAL PARAMETERS AND PHOTOMETRY
}

\author{
Genady Pilyavsky ${ }^{1}$, Suvrath Mahadevan ${ }^{1,2}$, Stephen R. Kane ${ }^{3}$, Andrew W. Howard ${ }^{4,5}$, David R. Ciardi ${ }^{3}$, \\ Chris de Pree ${ }^{6}$, Diana Dragomir ${ }^{3,7}$, Debra Fischer ${ }^{8}$, Gregory W. Henry ${ }^{9}$, Eric L. N. Jensen ${ }^{10}$, Gregory Laughlin ${ }^{11}$, \\ Hannah Marlowe ${ }^{6}$, Markus Rabus ${ }^{12}$, Kaspar von Braun ${ }^{3}$, Jason T. Wright ${ }^{1,2}$, and Xuesong X. Wang ${ }^{1}$ \\ ${ }^{1}$ Department of Astronomy and Astrophysics, Pennsylvania State University, 525 Davey Laboratory, University Park, PA 16802, USA; gcp5017@psu.edu \\ ${ }^{2}$ Center for Exoplanets \& Habitable Worlds, Pennsylvania State University, 525 Davey Laboratory, University Park, PA 16802, USA; suvrath@ astro.psu.edu \\ ${ }^{3}$ NASA Exoplanet Science Institute, Caltech, MS 100-22, 770 South Wilson Avenue, Pasadena, CA 91125, USA \\ ${ }_{5}^{4}$ Department of Astronomy, University of California, Berkeley, CA 94720, USA \\ ${ }^{5}$ Space Sciences Laboratory, University of California, Berkeley, CA 94720, USA \\ ${ }^{6}$ Department of Physics and Astronomy, Agnes Scott College, 141 East College Avenue, Decatur, GA 30030, USA \\ ${ }^{7}$ Department of Physics \& Astronomy, University of British Columbia, Vancouver, BC V6T1Z1, Canada \\ ${ }^{8}$ Department of Astronomy, Yale University, New Haven, CT 06511, USA \\ ${ }^{9}$ Center of Excellence in Information Systems, Tennessee State University, 3500 John A. Merritt Blvd., Box 9501, Nashville, TN 37209, USA \\ ${ }^{10}$ Department of Physics \& Astronomy, Swarthmore College, Swarthmore, PA 19081, USA \\ ${ }^{11}$ UCO/Lick Observatory, University of California, Santa Cruz, CA 95064, USA \\ ${ }^{12}$ Departamento de Astonomía y Astrofísica, Pontificia Universidad Católica de Chile, Casilla 306, Santiago 22, Chile \\ Received 2011 July 14; accepted 2011 September 21; published 2011 December 2
}

\begin{abstract}
The discovery of transiting planets around bright stars holds the potential to greatly enhance our understanding of planetary atmospheres. In this work we present the search for transits of HD 168443b, a massive planet orbiting the bright star HD $168443(V=6.92)$ with a period of 58.11 days. The high eccentricity of the planetary orbit $(e=0.53)$ significantly enhances the a priori transit probability beyond that expected for a circular orbit, making HD 168443 a candidate for our ongoing Transit Ephemeris Refinement and Monitoring Survey. Using additional radial velocities from Keck High Resolution Echelle Spectrometer, we refined the orbital parameters of this multiplanet system and derived a new transit ephemeris for HD 168443b. The reduced uncertainties in the transit window make a photometric transit search practicable. Photometric observations acquired during predicted transit windows were obtained on three nights. Cerro Tololo Inter-American Observatory $1.0 \mathrm{~m}$ photometry acquired on 2010 September 7 had the required precision to detect a transit but fell just outside of our final transit window. Nightly photometry from the T8 $0.8 \mathrm{~m}$ automated photometric telescope at Fairborn Observatory, acquired over a span of 109 nights, demonstrates that HD 168443 is constant on a timescale of weeks. Higher-cadence photometry on 2011 April 28 and June 25 shows no evidence of a transit. We are able to rule out a non-grazing transit of HD $168443 b$.
\end{abstract}

Key words: planetary systems - stars: individual (HD 168443) - techniques: photometric - techniques: radial velocities

Online-only material: color figures

\section{INTRODUCTION}

The number of known exoplanets has grown rapidly in the last decade, with over 600 confirmed exoplanets known to date. ${ }^{13}$ While most of these planets have been discovered using radial velocity (RV) techniques, the number of known transiting planets has increased significantly due to dedicated transit surveys like the space-based Kepler (Borucki et al. 2011) and CoRoT (Barge et al. 2008) missions, and ground-based transit searches like the Hungarian Automated Telescope Network (Bakos et al. 2004), SuperWASP (Pollacco et al. 2006), and $\mathrm{XO}$ (McCullough et al. 2005). The price for efficient operation of these wide-field transit surveys, though, is that most of the candidate stars tend to be fainter than those being surveyed by $\mathrm{RV}$. Of the over one hundred transiting planet host stars known, the sample of bright stars $(V<9)$ with transiting planets is still limited to only nine stars: HD 209458 (Charbonneau et al. 2000; Henry et al. 2000), HD 189733 (Bouchy et al. 2005), HD 149026 (Sato et al. 2005), HD 17156 (Barbieri et al. 2007), HD 80606 (Moutou et al. 2009; Fossey et al. 2009), HD 97658 (Henry et al. 2011), and 55 Cnc e (Winn et al. 2011), all of which were discovered by RV surveys, while WASP-33b and

\footnotetext{
13 http://exoplanet.eu/
}

HAT-P-2b were discovered by transit surveys and confirmed by RV follow-up.

The discovery of additional bright transiting planet hosts is advantageous in further enabling studies of the atmospheric constituents of exoplanets. Even with the largest ground-based telescopes, transmission spectroscopy to probe the atmospheres of these exoplanets has, largely, been accomplished only for the brightest targets. Using high-resolution spectroscopy on 8-10 m telescopes, Redfield et al. (2008) and Snellen et al. (2008) detected sodium absorption in the transmission spectra of HD189733b and HD209458b, respectively. More recently, using the narrowband tunable filter imager on the $10 \mathrm{~m}$ Gran Telescopio Canarias (GTC), Colon et al. (2010) and Sing et al. (2011) have detected the signature of potassium absorption in the atmospheres of HD 80606b and XO-2b.

Our ongoing Transit Ephemeris Refinement and Monitoring Survey (TERMS; Kane et al. 2009) project focuses on bright stars $(V<9)$ with known exoplanets and orbital periods greater than 10 days in an effort to refine the orbital parameters with additional RV observations and then observe the targets photometrically within their revised transit windows. Transits detected around such bright stars would provide perfect candidates for spectroscopic follow-up. In addition, with periods greater 
than 10 days, the planet population searched by TERMS is not easily duplicable by ongoing ground-based transit surveys as demonstrated by von Braun et al (2009). In Kane et al. (2011) we presented the ephemeris revision and the search for a transit around HD 156846. In this paper, we present additional RVs and refine the transit ephemeris for the bright star HD 168443, which is known to have multiple companions. We present new photometry that allows us to rule out transits of HD 168443b.

\section{HD 168443}

HD 168443 (GJ 4052, HIP 89844, and TYC 5681-1576-1) is a bright ( $V=6.92)$ G5 dwarf known to possess two substellar companions, forming a dynamically active system (Veras \& Armitage 2007). HD 168443b (Marcy et al. 1999; Wright et al. 2009) has a reported $M_{p} \sin i=7.8 \pm 0.259 M^{\mathrm{Jup}}$, an orbit with a period of 58.11 days, and a large eccentricity of $e=0.53$. HD 168443c, a brown dwarf companion (Udry et al. 2002; Wright et al. 2009), has $M_{p} \sin i=17.5 \pm 0.65 M^{\text {Jup }}$, an orbital period of $\sim 1748$ days, and a moderate eccentricity $e=0.21$. Using the van Leeuwen (2007) re-reduction of the Hipparcos data, Reffert \& Quirrenbach (2011) derive a mass of $30.3_{-12.2}^{+9.4} M^{\text {Jup }}$ and a $3 \sigma$ upper mass limit of $65 M^{\text {Jup }}$, confirming that this object is indeed substellar. The $3 \sigma$ lower limit does not exclude an inclination of $90^{\circ}$, so the minimum mass derived from the RVs applies. From CORALIE RVs and the van Leeuwen (2007) Hipparcos re-reductions, Sahlmann et al. (2011) concluded that, while their formal solution for the mass of HD 168443c matched that of Reffert \& Quirrenbach (2006), the mass was of "low confidence." They are unable to set an upper mass limit because the RV orbit is not fully covered by their CORALIE observations. Dynamical simulations by Veras $\&$ Ford (2010) show that almost no stable systems can exist for mutual inclinations between HD $168443 \mathrm{~b}$ and $\mathrm{c}$ of $60^{\circ}-120^{\circ}$.

The eccentric orbit of HD $168443 \mathrm{~b}$ increases its transit probability significantly above what one would expect for a planet in a circular orbit with the same period. The new orbital parameters, along with the formalism outlined in Kane et al. (2009), result in a transit probability of $3.7 \%$ compared to $2.5 \%$ for a circular orbit. While the atmospheric scale heights for massive, relatively cold planets are expected to be small (VidalMadjar et al. 2011), the brightness of HD 168443 (more than twice as bright as HD 209458, the third brightest star known to have a transiting planet) makes such detections possible with large ground-based telescopes.

The discovery of transits of the inner planet would also constrain the possible inclinations of the outer brown dwarf companion, enabling additional dynamical investigations. The predicted transit probability of $3.7 \%$, coupled with the fact that HD 168443 is a very bright star, makes it an intriguing target in our ongoing attempts to discover long-period transiting planets.

\section{STELLAR PROPERTIES}

We used Spectroscopy Made Easy (Valenti \& Piskunov 1996) to fit high-resolution Keck High Resolution Echelle Spectrometer (HIRES) spectra of HD 168443, applying the wavelength intervals, line data, and methodology of Valenti \& Fischer (2005). We further constrained the surface gravity using Yonsei-Yale $\left(\mathrm{Y}^{2}\right)$ stellar structure models (Demarque et al. 2004) and revised Hipparcos parallaxes (van Leeuwen 2007), with the iterative method of Valenti et al. (2009). The resulting stellar parameters listed in Table 1 are effective temperature, surface gravity, iron abundance, projected rotational velocity, mass, and radius.
Table 1

Stellar Properties

\begin{tabular}{lcl}
\hline \hline Parameter & Value & \multicolumn{1}{c}{ Reference } \\
\hline$M_{V}$ & 4.198 & van Leeuwen $(2007)$ \\
$B-V$ & 0.724 & Bessell (2000) \\
$V$ & 6.92 & Bessell (2000) \\
Distance $(\mathrm{pc})$ & $37.4 \pm 1.0$ & van Leeuwen $(2007)$ \\
$T_{\mathrm{eff}}(\mathrm{K})$ & $5491 \pm 44$ & This work \\
$\log g$ & $4.07 \pm 0.06$ & This work \\
{$[\mathrm{Fe} / \mathrm{H}]$} & $+0.04 \pm 0.03$ & This work \\
$v \sin i\left(\mathrm{~km} \mathrm{~s}^{-1}\right)$ & $2.20 \pm 0.50$ & This work \\
$M_{\star}\left(M_{\odot}\right)$ & $0.995 \pm 0.019$ & This work \\
$R_{\star}\left(R_{\odot}\right)$ & $1.51 \pm 0.06$ & This work \\
$\log R_{\mathrm{HK}}^{\prime}$ & -5.088 & This work \\
$S_{\mathrm{HK}}$ & 0.148 & This work \\
\hline
\end{tabular}

HD 168443 lies 1.05 mag above the Hipparcos average main sequence $\left(M_{V}\right.$ versus $\left.B-V\right)$ as defined by Wright (2005). These properties are consistent with an evolved metal-rich G5 star. The stellar radius, $R_{\star}=1.51 \pm 0.06 R_{\odot}$, is crucial for estimating the depth and duration of a planetary transit. This value is consistent with the interferometrically measured $R_{\star}=1.58 \pm 0.06 R_{\odot}$ (van Belle \& von Braun 2009).

In addition, we computed the level of stellar activity in HD 168443 from the strength of the Ca II H \& K lines, which give calibrated $S_{\mathrm{HK}}$ values on the Mt. Wilson scale and $\log R_{\mathrm{HK}}^{\prime}$ values (Isaacson \& Fischer 2010). The median of $\log R_{\mathrm{HK}}^{\prime}$ and $S_{\mathrm{HK}}$ values are listed in Table 1 and demonstrate that HD 168443 is chromospherically quiet. Additional examination of the available history of Ca II $\mathrm{H} \& \mathrm{~K}$ measurements shows no significant long-term variation in $S_{\mathrm{HK}}$.

\section{KECK-HIRES RV MEASUREMENTS AND REVISED ORBITAL MODEL}

\subsection{Measurements}

We observed HD 168443 using the standard procedures of the California Planet Search for the HIRES echelle spectrometer (Vogt et al. 1994) on the $10 \mathrm{~m}$ Keck I telescope. These measurements span 15 years, 1996 July to 2011 March, and comprise one of the longest RV data sets presented for a star with one or more known planets. The initial measurements in this time series were used to discover the two planets (Marcy et al. 1999, 2001) while later measurements refined the orbits (Wright et al. 2009). The full set of measurements presented here refines the orbit further and gives an accurate predicted transit ephemeris with which we search for photometric transits.

The 130 Keck RV measurements (Table 2) were made from observations with an iodine cell mounted directly in front of the spectrometer entrance slit. The dense set of molecular absorption lines imprinted on the stellar spectra provide a robust wavelength fiducial against which Doppler shifts are measured, as well as strong constraints on the shape of the spectrometer instrumental profile at the time of each observation (Marcy \& Butler 1992; Valenti et al. 1995). We measured the Doppler shift of each star-times-iodine spectrum using a modeling procedure descended from Butler et al. (1996) as described in Howard et al. (2009). The times of observation (in barycentric Julian days), relative RVs, and associated errors (excluding jitter) are listed in Table 2. We also observed HD 168443 with the iodine cell removed to construct a stellar template spectrum for Doppler modeling and to derive stellar properties. 
Table 2

Keck Radial Velocities

\begin{tabular}{|c|c|c|c|c|c|}
\hline $\mathrm{BJD}-2,440,000$ & $\begin{array}{l}\text { Radial Velocity } \\
\left(\mathrm{m} \mathrm{s}^{-1}\right)\end{array}$ & $\begin{array}{l}\text { Uncertainty } \\
\left(\mathrm{m} \mathrm{s}^{-1}\right)\end{array}$ & $\overline{\mathrm{BJD}}-2,440,000$ & $\begin{array}{l}\text { Radial Velocity } \\
\left(\mathrm{m} \mathrm{s}^{-1}\right)\end{array}$ & $\begin{array}{c}\text { Uncertainty } \\
\left(\mathrm{m} \mathrm{s}^{-1}\right)\end{array}$ \\
\hline 10276.90890 & -309.29 & 1.73 & 11883.68272 & 498.30 & 0.86 \\
\hline 10603.01184 & -33.47 & 1.02 & 11983.15885 & 333.08 & 1.01 \\
\hline 10665.86781 & -69.33 & 1.24 & 11984.15396 & 335.98 & 1.35 \\
\hline 10713.73770 & -73.02 & 1.14 & 12004.12317 & 357.01 & 1.33 \\
\hline 10714.76649 & -71.38 & 1.13 & 12005.14737 & 352.21 & 1.37 \\
\hline 10955.01039 & -5.57 & 1.12 & 12007.13381 & 329.80 & 1.16 \\
\hline 10955.95862 & -5.13 & 1.02 & 12008.03597 & 311.14 & 1.37 \\
\hline 10957.07105 & -4.68 & 1.05 & 12009.10491 & 294.16 & 1.24 \\
\hline 10981.88012 & -568.69 & 1.01 & 12030.98196 & -27.97 & 1.26 \\
\hline 10982.89132 & -492.38 & 1.10 & 12061.94969 & 278.32 & 1.37 \\
\hline 10983.07690 & -472.56 & 1.10 & 12062.96034 & 259.31 & 1.54 \\
\hline 10983.82231 & -420.40 & 1.16 & 12094.88634 & 78.37 & 1.40 \\
\hline 10984.06138 & -407.63 & 1.14 & 12096.93360 & 110.11 & 1.52 \\
\hline 11009.87009 & 22.04 & 1.34 & 12098.01419 & 126.96 & 1.38 \\
\hline 11010.05994 & 24.94 & 0.93 & 12099.02269 & 145.64 & 1.34 \\
\hline 11010.85123 & 20.67 & 1.17 & 12099.93319 & 147.47 & 1.23 \\
\hline 11011.86077 & 25.19 & 1.37 & 12100.94388 & 158.52 & 1.41 \\
\hline 11012.95413 & 19.19 & 1.14 & 12101.88510 & 171.72 & 1.42 \\
\hline 11013.06816 & 20.31 & 0.82 & 12127.86347 & 57.89 & 1.51 \\
\hline 11013.82791 & 15.69 & 1.15 & 12128.79403 & 30.56 & 1.23 \\
\hline 11013.92983 & 15.33 & 1.20 & 12133.78671 & -210.61 & 1.53 \\
\hline 11042.95557 & -326.88 & 1.09 & 12160.80699 & 79.11 & 1.48 \\
\hline 11043.95602 & -276.32 & 1.19 & 12189.76875 & -156.78 & 1.43 \\
\hline 11050.81406 & -77.68 & 1.26 & 12445.93556 & -192.60 & 1.38 \\
\hline 11068.77042 & 54.21 & 1.08 & 12486.81165 & -930.27 & 1.38 \\
\hline 11069.78596 & 60.86 & 1.09 & 12515.75755 & -85.29 & 1.44 \\
\hline 11070.79807 & 52.81 & 1.09 & 12536.74799 & -258.80 & 1.39 \\
\hline 11071.76998 & 50.98 & 1.11 & 12572.69122 & -74.58 & 1.31 \\
\hline 11072.76271 & 47.77 & 1.26 & 12713.14689 & -280.58 & 1.33 \\
\hline 11074.78514 & 39.07 & 1.09 & 12778.02605 & -891.12 & 1.36 \\
\hline 11228.16111 & 91.81 & 1.07 & 12804.06082 & 12.02 & 1.25 \\
\hline 11229.14942 & 95.62 & 1.32 & 12834.88152 & -730.70 & 1.31 \\
\hline 11311.04174 & 151.35 & 1.27 & 12848.80396 & -133.69 & 1.29 \\
\hline 11312.07757 & 137.66 & 1.23 & 12855.96590 & 0.22 & 1.28 \\
\hline 11313.07589 & 124.69 & 1.18 & 12898.71740 & -579.54 & 1.16 \\
\hline 11314.08699 & 103.43 & 1.20 & 13154.04176 & 260.17 & 1.49 \\
\hline 11341.02661 & 109.66 & 1.11 & 13180.90222 & -183.23 & 1.37 \\
\hline 11341.90588 & 129.71 & 1.03 & 13195.84965 & 58.60 & 1.13 \\
\hline 11342.97144 & 142.29 & 1.20 & 13238.88150 & -123.80 & 1.17 \\
\hline 11367.86065 & 214.31 & 1.30 & 13301.73743 & -565.16 & 1.16 \\
\hline 11368.84314 & 205.20 & 1.40 & 13546.89064 & 334.18 & 1.14 \\
\hline 11370.01150 & 188.83 & 1.14 & 13842.12630 & 125.12 & 1.18 \\
\hline 11370.91933 & 171.14 & 0.73 & 13927.87788 & -3.76 & 1.15 \\
\hline 11371.91024 & 153.12 & 1.11 & 13984.83683 & -51.21 & 0.96 \\
\hline 11372.87811 & 131.18 & 1.16 & 14314.99539 & -101.88 & 1.26 \\
\hline 11373.79496 & 105.31 & 1.44 & 14335.95562 & -191.79 & 1.23 \\
\hline 11409.85005 & 276.28 & 1.14 & 14343.88345 & -620.98 & 1.00 \\
\hline 11410.84584 & 278.53 & 1.29 & 14344.94159 & -744.93 & 1.11 \\
\hline 11411.84641 & 288.70 & 1.21 & 14398.74642 & -344.38 & 1.08 \\
\hline 11438.73808 & -282.82 & 1.19 & 14546.11545 & -16.53 & 1.17 \\
\hline 11439.73057 & -414.58 & 1.18 & 14548.15404 & 10.39 & 1.27 \\
\hline 11440.71830 & -527.69 & 1.22 & 14720.84072 & 102.86 & 1.11 \\
\hline 11441.73997 & -616.71 & 1.20 & 14956.12833 & 289.18 & 1.53 \\
\hline 11679.04801 & -109.80 & 1.09 & 14985.11014 & -473.97 & 1.33 \\
\hline 11680.07181 & -20.27 & 1.29 & 15014.97707 & 332.37 & 1.13 \\
\hline 11703.01759 & 488.68 & 1.19 & 15026.96186 & 318.51 & 1.22 \\
\hline 11703.98840 & 488.90 & 1.17 & 15106.74809 & -279.16 & 1.20 \\
\hline 11705.03899 & 493.45 & 1.27 & 15286.11499 & 173.04 & 1.21 \\
\hline 11705.95846 & 499.46 & 1.30 & 15322.08481 & 403.90 & 1.21 \\
\hline 11707.08125 & 502.77 & 1.20 & 15343.00253 & 103.85 & 1.36 \\
\hline 11754.85883 & 473.14 & 1.66 & 15374.84199 & 453.14 & 1.16 \\
\hline 11755.90986 & 489.51 & 1.06 & 15378.82083 & 413.14 & 1.21 \\
\hline 11792.74760 & -338.38 & 1.01 & 15403.81739 & 198.38 & 1.22 \\
\hline 11793.80008 & -223.10 & 1.25 & 15490.73506 & 346.47 & 1.30 \\
\hline 11882.68333 & 496.02 & 0.88 & 15636.13540 & -128.87 & 1.05 \\
\hline
\end{tabular}

Table 2

(Continued) 
Table 3

Keplerian Orbital Model

\begin{tabular}{|c|c|}
\hline Parameter & Value \\
\hline \multicolumn{2}{|l|}{ HD $168443 b$} \\
\hline$P$ (days) & $58.11247 \pm 0.0003$ \\
\hline$T_{c}^{\mathrm{a}}(\mathrm{JD}-2,440,000)$ & $15621.637 \pm 0.0156$ \\
\hline$T_{p}^{\mathrm{b}}(\mathrm{JD}-2,440,000)$ & $15626.199 \pm 0.024$ \\
\hline$e$ & $0.52883 \pm 0.00103$ \\
\hline$K\left(\mathrm{~m} \mathrm{~s}^{-1}\right)$ & $475.133 \pm 0.9102$ \\
\hline$\omega(\mathrm{deg})$ & $172.923 \pm 0.139$ \\
\hline$M \sin i\left(M^{\mathrm{Jup}}\right)$ & $7.659 \pm 0.0975$ \\
\hline$a(\mathrm{AU})$ & $0.2931 \pm 0.00181$ \\
\hline \multicolumn{2}{|l|}{ HD $168443 c$} \\
\hline$P$ (days) & $1749.83 \pm 0.57$ \\
\hline$T_{c}^{\mathrm{a}}(\mathrm{JD}-2,440,000)$ & $15599.9 \pm 1.187$ \\
\hline$T_{p}^{\mathrm{b}}(\mathrm{JD}-2,440,000)$ & $15521.3 \pm 2.2$ \\
\hline$e$ & $0.2113 \pm 0.00171$ \\
\hline$K\left(\mathrm{~m} \mathrm{~s}^{-1}\right)$ & $297.70 \pm 0.618$ \\
\hline$\omega(\mathrm{deg})$ & $64.87 \pm 0.5113$ \\
\hline$M \sin i\left(M^{\mathrm{Jup}}\right)$ & $17.193 \pm 0.21$ \\
\hline$a(\mathrm{AU})$ & $2.8373 \pm 0.018$ \\
\hline \multicolumn{2}{|l|}{ System Properties } \\
\hline$\gamma\left(\mathrm{m} \mathrm{s}^{-1}\right)$ & $-46.533 \pm 0.552$ \\
\hline$d v / d t\left(\mathrm{~m} \mathrm{~s}^{-1} \mathrm{yr}^{-1}\right)$ & $-0.00868 \pm 0.00025$ \\
\hline \multicolumn{2}{|l|}{ Measurements and Model } \\
\hline$N_{\text {obs }}$ & 130 \\
\hline $\mathrm{rms}\left(\mathrm{m} \mathrm{s}^{-1}\right)$ & 3.90 \\
\hline$\chi_{v}^{2}$ & 1.44 \\
\hline
\end{tabular}

Notes.

a Time of transit.

b Time of periastron passage.

\subsection{Keplerian Model}

We modeled the Keck RVs as the superposition of the Keplerian interactions from two planets with the star, plus a linear trend in velocity due to a distant and massive third companion. We used the orbit-fitting techniques described in Howard et al. (2010) and the partially linearized, least-squares fitting procedure described in Wright \& Howard (2009). Each velocity measurement was assigned a weight, $w$, constructed from the quadrature sum of the measurement uncertainty $\left(\sigma_{\mathrm{RV}}\right)$ and a jitter term $\left(\sigma_{\text {jitter }}\right)$, i.e., $w=1 /\left(\sigma_{\mathrm{RV}}^{2}+\sigma_{\text {jitter }}^{2}\right)$. We chose jitter values of $\sigma_{\text {jitter }}=3$ and $2 \mathrm{~m} \mathrm{~s}^{-1}$ for measurements before and after the HIRES upgrade in 2004 August. These values are consistent with the expected jitter of a slightly evolved early G star observed with Keck-HIRES (Wright 2005). Possible sources of jitter include stellar pulsation, magnetic cycles, granulation, undetected planets, and instrumental effects (Isaacson \& Fischer 2010; Wright 2005).

Our best-fit orbital model is presented in Table 3 and Figure 1. The Keplerian parameter uncertainties for each planet were derived using a Monte Carlo method (Marcy et al. 2005) and account for correlations between parameter errors. Uncertainties in $M \sin i$ and $a$ reflect uncertainties in $M_{\star}$ and the orbital parameters. We considered and rejected more complicated models having a third planet and/or a quadratic velocity trend because of statistically insignificant changes in $\chi^{2}$ compared to the adopted model (Table 3 ).

\section{TRANSIT EPHEMERIS REFINEMENT}

The revised orbital solution presented in Table 3 for this multiplanet system together with the stellar properties in Table 1 allow us to construct an accurate transit ephemeris from which
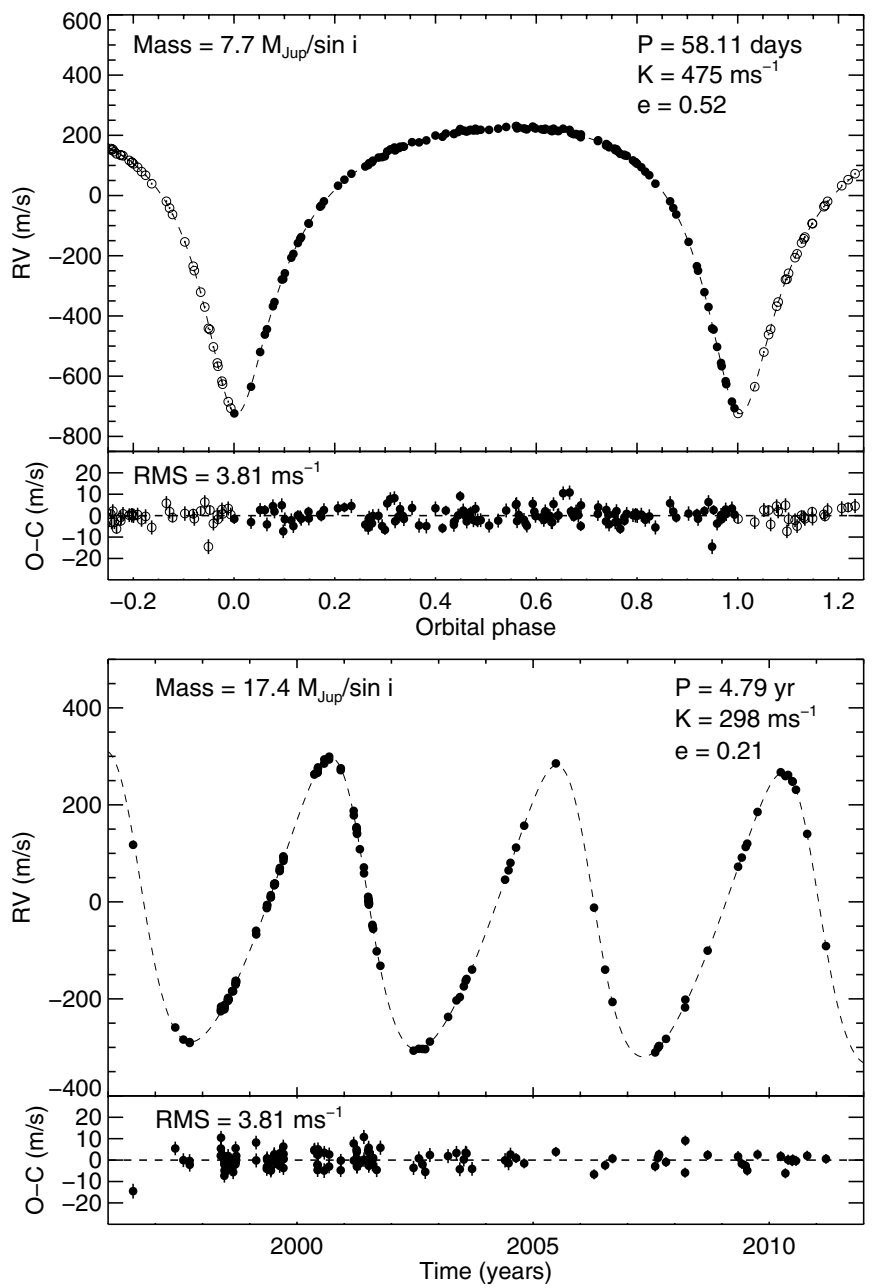

Figure 1. RV measurements of HD 168443 from Keck-HIRES (filled circles) with the Keplerian orbital model (dashed lines). The top panel shows the RVs phased to the orbital period of HD $168443 \mathrm{~b}$ with the model for the other planet and linear trend subtracted. Open circles represent the same RV measurements wrapped one orbital phase. The bottom panel shows the RV time series illustrating the variations due to HD $168443 \mathrm{c}$, with the linear velocity trend and the orbit of HD 168443 bubtracted.

to conduct a search for transits. As shown by Kane \& von Braun (2008), the transit probability of a planet is intricately related to both the orbital eccentricity and the argument of periastron. Figure 2 depicts the orbits of the planets relative to the observer line of sight and shows how the eccentricities and orientations affects the star-planet separation along that line.

We use the models of Bodenheimer et al. (2003) to estimate a radius for HD $168443 \mathrm{~b}$ of $R_{p}=1.11 R^{\mathrm{Jup}}$, which takes into account orbital parameters and the stellar flux received by the planet. This results in a transit probability of $3.7 \%$ and a predicted transit depth of $0.6 \%$. The predicted transit duration is 0.36 days, or $8 \mathrm{hr}$ and 40 minutes. The time of mid transit shown in Table 3 is for 2011 March 1. The calculation was performed using a Monte Carlo bootstrap which propagates the uncertainties in the orbital parameters forward to the time of transit. The uncertainty in this predicted time is small, only $\sim 35$ minutes. Thus, the predicted duration of the transit window for this date is $9 \mathrm{hr}$ and 50 minutes (the sum of the predicted duration $\pm 1 \sigma$ deviation), overwhelmingly dominated by the predicted transit duration rather than the uncertainty associated with the orbital parameters. The predicted duration 


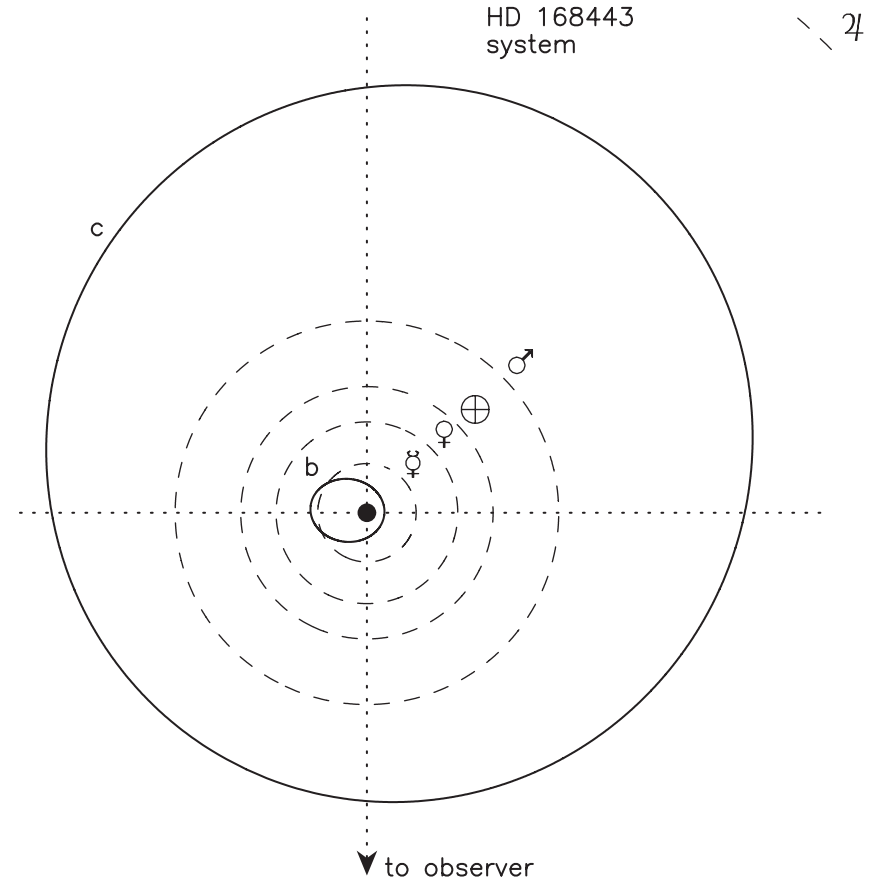

Figure 2. Orbits of the planets HD 168443b and HD 168443c shown in solid lines. Orbits of Mercury (eccentricity set to zero for clarity) through Jupiter are represented with dashed ovals.

makes complete coverage of the transit window from a single ground-based longitude difficult, though the predicted depth is sufficient to rule out transits from observations during times of ingress or egress only.

\section{HIPPARCOS PHOTOMETRY OF HD 168443}

The Hipparcos mission observed the brightest stars in the sky over many epochs. Robichon \& Arenou (2000) and Castellano et al. (2000) detected the transit of HD 209458b in the Hipparcos epoch photometry, and Hébrard \& Lecavelier Des Etangs (2006) were able to detect multiple transits of HD 189733b, leading to a significant improvement in the determination of its period. Hipparcos photometry has therefore been demonstrated to be precise enough to detect the transit of a hot Jupiter around stars that are fainter than HD $168443(V=6.92)$. The top panels of Figure 3 show the Hipparcos photometry of HD 209458 plotted against Julian Date and also against orbital phase of the star's hot-Jupiter companion. A few observations fall within the modern transit window and show a clear dimming. The bottom two panels are similar plots for HD 168443. Using our new RV observations, we followed the prescription of Robichon \& Arenou (2000) to create the phased plot with the predicted transit set at zero phase. Three Hipparcos photometric observations acquired around BJD 2448050 lie inside of our predicted transit window. Unlike the case for HD 209458, the Hipparcos photometry show no evidence for a transit. However, the expected transit depth for HD $168443 \mathrm{~b}$ is significantly smaller than HD 209458; the Hipparcos photometry alone would have been an unreliable guide to prove or disprove the occurrence of transits in HD 168443. Nevertheless, we advocate such a check for bright stars with sufficiently precise orbital ephemerides.
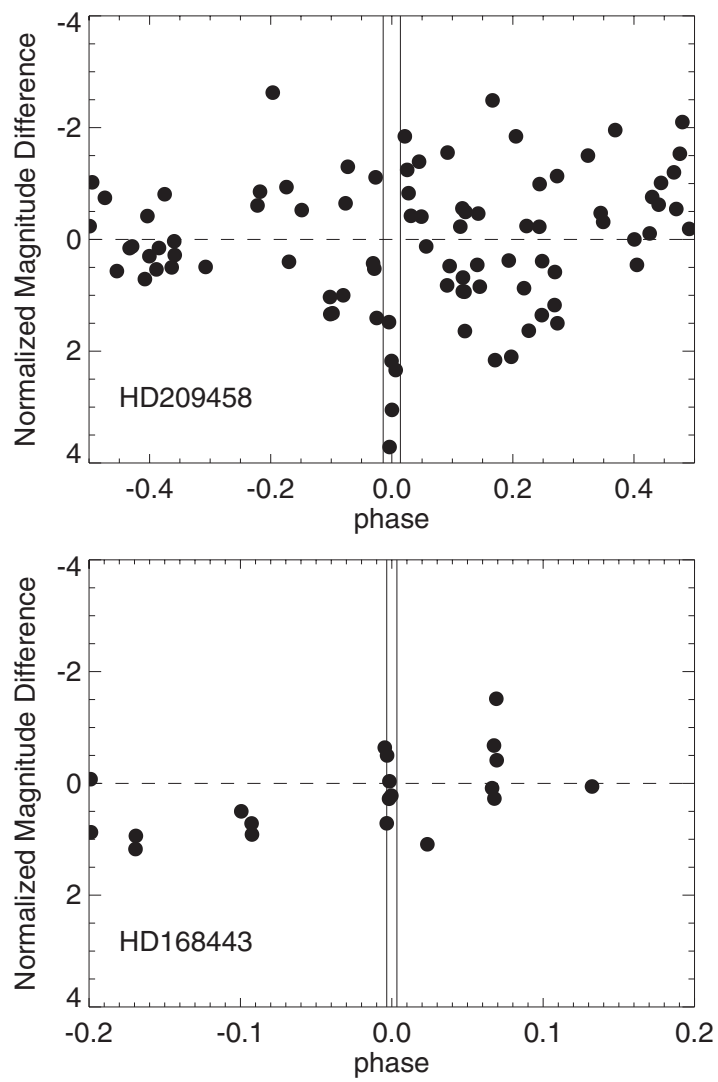

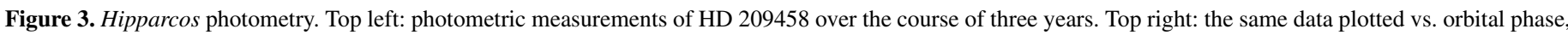

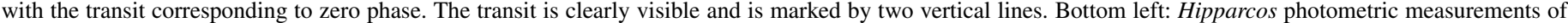

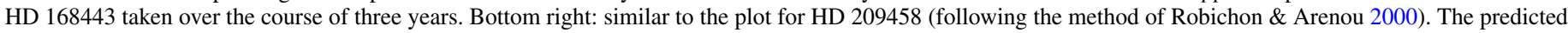
window is bounded by two vertical lines, with three Hipparcos measurements obtained during the predicted transit. No transit is evident. 


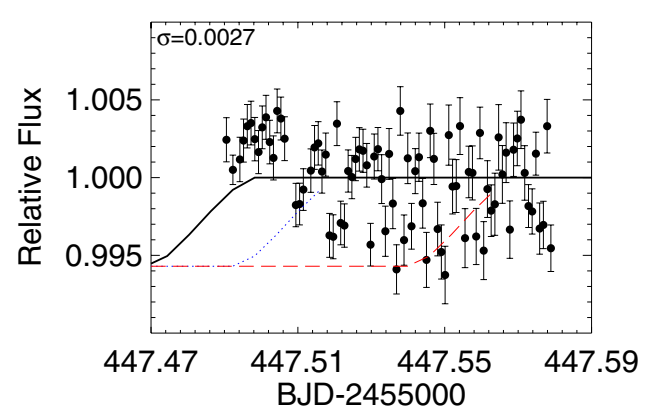

Figure 4. Relative photometry from CTIO. The standard deviation of 0.0027 in the normalized flux is precise enough to detect the predicted transit, which would causes a 0.006 decrease. The solid line represents the predicted transit after ephemeris refinement using Keck-HIRES data, with the predicted egress occurring right before the measurements were acquired. The dotted blue and the dashed red lines represent the $1 \sigma$ and $3 \sigma$ uncertainties in the time of egress. (A color version of this figure is available in the online journal.)

\section{PHOTOMETRY OF THE REVISED TRANSIT WINDOW}

In addition to the new TERMS photometry presented below, we also observed HD 168443 with the Southeastern Association for Research in Astronomy (SARA) $0.6 \mathrm{~m}$ telescope at Cerro Toledo Inter-American Observatory (CTIO). However, due to poor weather conditions, the SARA-S measurements exhibited scatter that was significantly higher than the predicted transit depth. Therefore, we do not discuss these data further, but we note that planning such multi-site observations is necessary in our TERMS search for long-period transits.

\subsection{Cerro Tololo Inter-American Observatory}

Before we had access to the latest Keck-HIRES RVs, we used published orbital parameters (Wright et al. 2009) to calculate the transit ephemeris and to schedule an observing run for 2010 September 7 with the CTIO $1.0 \mathrm{~m}$ telescope and Y4KCam CCD detector. The observations were made through a Johnson-Morgan $R$-band filter; instrumental magnitudes of HD 168443 and comparison stars were extracted from the images with an IDL implementation of DAOPHOT (Stetson 1987). Relative fluxes (Everett \& Howell 2001) of HD 168443 were computed with respect to the two stable comparison stars (TYC 5681-1450-1 and TYC 5681-1458-1).

The results are plotted in Figure 4. The solid curve represents the predicted transit fluxes computed from our new orbital elements in Table 2, assuming on-time, central transits with a predicted depth of $0.6 \%$ or 0.006 flux units. The scatter of the measurements is 0.0027 , easily sufficient to detect the predicted transits. However, these measurements cover only the later part of the predicted transit window; an egress late by $1 \sigma$ and $3 \sigma$ are represented with dashed blue and red lines, respectively. The CTIO data show only that late transits do not occur.

We have presented these measurements because CTIO photometric monitoring is an essential component of the TERMS strategy. Had the ephemerides (based on orbital parameters in the literature) been more precise, the CTIO photometry has the requisite precision to detect a predicted on-time transit. Our results demonstrate the precision achievable from CTIO with a typical TERMS target.

\subsection{Fairborn Observatory}

We obtained additional photometric observations of HD 168443 with the T8 0.8 m automatic photometric telescope (APT) at Fairborn Observatory in southern Arizona. The T8

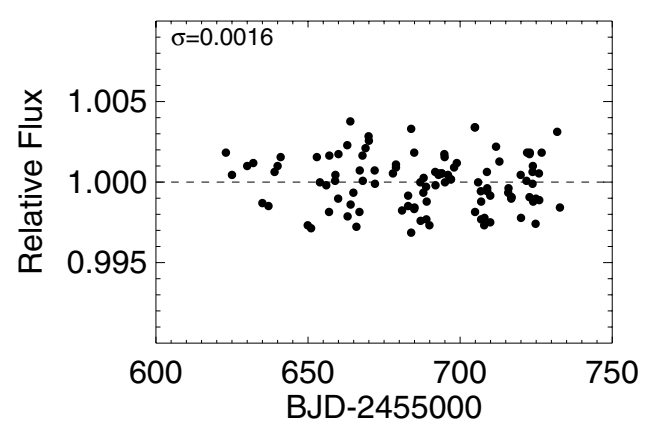

Figure 5. Photometry from the T8 APT consisting of 107 measurements over the span of 109 days. The dashed line represents the normalized flux. The standard deviation from the mean is 0.0016 .

APT uses a two-channel precision photometer with two EMI 9124QB bi-alkali photomultiplier tubes to make simultaneous measurements in the Strömgren $b$ and $y$ passbands. The telescope was programmed to make nightly differential brightness measurements of HD 168443 with respect to the comparison star HD 166664. Three consecutive differential measurements were co-added to create a single nightly differential magnitude. To improve the precision of these brightness measurements, we combined the individual $b$ and $y$ differential magnitudes into a mean $(b+y) / 2$ "passband." The typical precision of a single observation on good nights is $\sim 0.0015$ mag; see Henry (1999) for further details on telescope design and operations, data reduction, calibrations, and data precision.

Between 2011 March 2 and June 19, the APT collected 107 nightly observations of HD 168443 with respect to HD 166664; the differential $(b+y) / 2$ magnitudes were converted to relative fluxes and are plotted in Figure 5. The nightly observations scatter about their mean flux, indicated by the dashed line in Figure 5, with a standard deviation of 0.0016 , which is consistent with constant stars. Periodogram analyses from 1 to 100 days reveal no significant periodicity. We conclude that HD 168443 is constant on its rotation timescale. A least-squares sine fit of the nightly observations on the orbital period of $168443 \mathrm{~b}$ gives a semi-amplitude of $0.00026 \pm 0.00023$ flux units. This very low limit to brightness variability in HD 168443 on the 58 day orbital period indicates that rotational modulation of starspots is not the cause of the RV variations (see, e.g., Queloz et al. 2001).

We also used the T8 APT to monitor HD 168443 on two nights in the 2011 observing season when our new ephemeris predicted additional transit events. Each monitoring observation consists of a single differential measurement rather than the mean of three observations, as we used for the nightly observations; thus the monitoring observations will have more scatter than the nightly observations.

On 2011 April 28 UT, an observable egress was to occur at BJD 2,455,679.937. We successfully monitored the star and obtained the 80 measurements plotted in Figure 6. The star was still two months before its opposition, so the start time was delayed until the star rose above an airmass of $\sim 2.0$; the observations ended $3.3 \mathrm{hr}$ later (at dawn). The sudden increase of the scatter after predicted egress is the result of a plume of smoke from one of the many wildfires burning throughout southern Arizona at the time. The standard deviation of the entire data set is 0.0040 flux units. The solid curve in Figure 6 represents the predicted fluxes for an on-time, central transit. The dotted blue lines represent the $\pm 1 \sigma$ uncertainty in the predicted time of central transit. The difference between the mean flux of the 


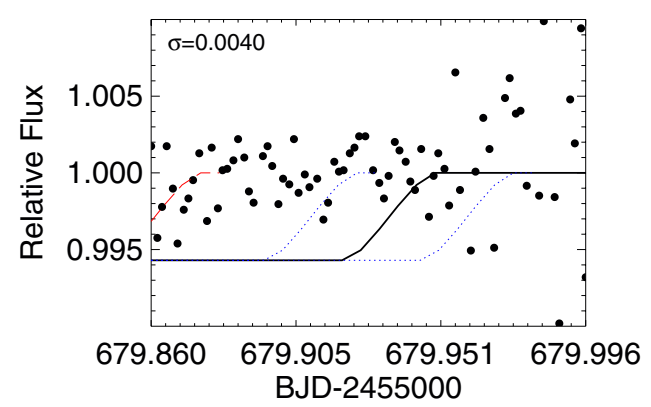

Figure 6. Photometric observations of HD 168443 acquired with the T8 APT at Fairborn Observatory during the predicted transit of 2011 April 28. The standard deviation of 0.0040 flux units is sufficient to detect the predicted transit egress (solid line) if present. The dotted blue and the dashed red lines represent $\pm 1 \sigma$ and $\pm 3 \sigma$ deviations in the time of transit, respectively. There is no evidence for an egress event.

(A color version of this figure is available in the online journal.)

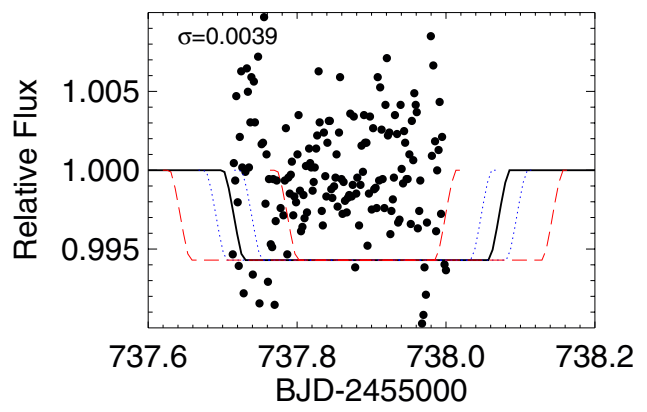

Figure 7. Photometric observations of HD 168443 with the T8 APT during the predicted transit of 2011 June 25 . The standard deviation of 0.0039 flux units is sufficient to detect the predicted transit egress (solid line) if present. The dotted blue and the dashed red lines represent $\pm 1 \sigma$ and $\pm 3 \sigma$ deviations in the time of transit, respectively. Again, there is no indication of a transit.

(A color version of this figure is available in the online journal.)

47 pre-egress observations and the 33 post-egress observations is only $0.0005 \pm 0.0011$. Given the tight limit on the transit time (35 minutes or 0.024 days), these observations rule out central transits with the predicted depth of 0.006 with a signal-to-noise ratio $(\mathrm{S} / \mathrm{N})$ of $\sim 5: 1$.

The next predicted transit was calculated to occur 58 days later on 2011 June 25 UT, centered at BJD 2,455,737.862. HD 168443 was at opposition during this transit, so we were able to acquire 165 observations over an interval of $7.1 \mathrm{hr}$. The declination of HD 168443 is approximately $-10^{\circ}$, so the airmass values at the start and at the end of the night were 2.25 and 2.51, respectively, for the east and west observing limits. Atmospheric extinction was also significantly higher than normal at the beginning of the night due to airborne dust, which gradually settled out over the course of the night. Therefore, in addition to reducing the transit observations with larger than normal extinction coefficients, we also removed a linear trend of approximately 0.008 flux units via a linear least-squares fit. The residuals from the line fit are plotted in Figure 7, again compared with predicted transit fluxes. The overall scatter of the 165 observations is 0.0039 flux units.

Finally, we estimate an upper limit to the possible transits of HD 168443b. The mean differential magnitudes of the T8 nightly observations in Figure 5 and of the mean of the first and second T8 transit monitoring observations in Figures 6 and 7 , before they were converted to relative fluxes, are $-0.24041 \pm 0.00017 \mathrm{mag},-0.23961 \pm 0.00050 \mathrm{mag}$, and $-0.24044 \pm 0.00037 \mathrm{mag}$, respectively. So, the observed difference between the mean of the nightly observations and the

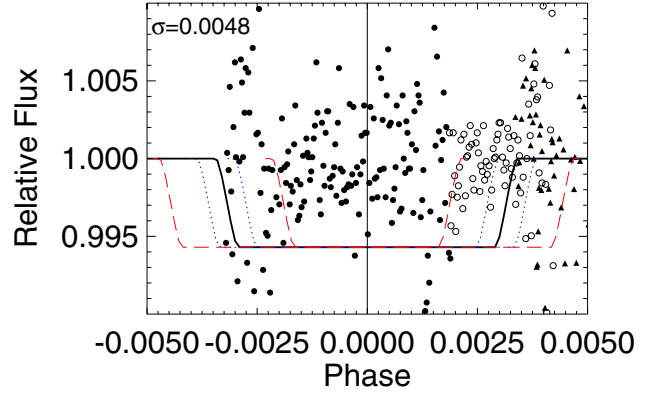

Figure 8. Phase diagram of the observations from all three nights of transit monitoring. The filled and unfilled circles are the T8 APT measurements from 2011 June 25 and April 28, respectively. The filled triangles are the CTIO observations from 2010 September 7. As in earlier figures, the solid line represents the predicted flux changes during a transit. The dotted blue and the dashed red lines represent $\pm 1 \sigma$ and $\pm 3 \sigma$ deviations in the time of transit, respectively.

(A color version of this figure is available in the online journal.)

mean of the transit observations is only $0.00038 \pm 0.00045 \mathrm{mag}$ or $0.00035 \pm 0.00041$ flux units. Therefore, we should have been able to detect a transit depth of $0.2 \%$ or 0.002 at an $\mathrm{S} / \mathrm{N}$ of almost 5:1, but our observations show no evidence of a transit.

\subsection{Full Coverage of the Transit Window}

The entire transit window was covered during the three monitoring nights. Initially, we were able to rule out only a late egress using $1 \mathrm{~m}$ CTIO photometry from 2010 September 8 with $(3 \sigma)$ confidence. We further confirmed this result with the APT photometry on June 25 by ruling out a late ingress $(3 \sigma)$. The early $(1 \sigma)$ and on-time egress was ruled out with APT photometry from 2011 April 28. In Figure 8, we present a phase plot containing all the monitoring nights. Since both data sets from the T8 APT used the same reference star, we placed the median of the measurements on the same $y$-axis scale. The CTIO data set was adjusted with an offset using the overlapping points from CTIO and the APT. The center of predicted transit is at phase 0.0 , marked by a solid vertical line. The dotted blue lines represent $1 \sigma$ early and late windows, while the dashed red lines represent $3 \sigma$ deviation from the center of the predicted transit.

\section{DISCUSSION}

As part of our ongoing TERMS, we present revised orbital parameters for the HD 168443 system, based on $130 \mathrm{RV}$ measurements with Keck-HIRES that span almost 15 years. Using the transit ephemerides derived from the revised orbital parameters, we searched for a transit using telescopes from CTIO, Fairborn Observatory, and SARA-S. We find no evidence of a detectable transit. The presence of a non-grazing transit corresponding to our model (1.1 $R^{\text {Jup }}$ planet $)$ is ruled out at a high level of confidence with high-precision photometry acquired by the APT. Grazing transits or transits with a planet radii as small as $0.58 R^{\text {Jup }}$ (which would yield densities much too high) are formally excluded at $1 \sigma$ confidence, though the smaller duration of such transits implies that the time of our photometric observations could have missed the ingress and egress. Using our orbital solution, and the planetary and stellar radii presented in this paper, we derive an upper limit on inclination of the system at 87.8 using methods described by Kane \& von Braun (2008). 
Even with a number of RV observations, determining precise orbital parameters for one component of a multi-planet system can be difficult. The recent discovery of transits of $55 \mathrm{Cnc}$ e by Winn et al. (2011), based on a new orbital period by Dawson \& Fabrycky (2010), illustrates the insidious impact of aliases and harmonics. Nevertheless, these effects can be mitigated by careful observation and analysis, and the value of additional precision RVs and revised orbital parameters cannot be overstated.

While we do not see transits in the HD 168443 system, the experimental approach outlined here, combining high-precision RV with multiple photometric telescope facilities, is worth pursuing in the quest for new transiting planets around bright nearby stars.

This work made use of the SIMBAD database (operated at CDS, Strasbourg, France), NASA's Astrophysics Data System Bibliographic Services, and the NASA Star and Exoplanet Database (NStED). This work was partially supported by funding from the Center for Exoplanets and Habitable Worlds, supported by the Pennsylvania State University, the Eberly College of Science, and the Pennsylvania Space Grant Consortium. The authors thank Andrés Jordán for providing support for the observations at CTIO. G.W.H. acknowledges support from NASA, NSF, Tennessee State University, and the Tennessee Centers of Excellence Program. E.L.N.J. acknowledges support from NSF Grant AST-0721386. M.R. acknowledges support from ALMACONICYT projects 31090015 and 31080021 . We thank the referee for insightful comments that helped us to improve this paper. Finally, the authors wish to extend special thanks to those of Hawai 'ian ancestry on whose sacred mountain of Mauna Kea we are privileged to be guests. Without their generous hospitality, the Keck observations presented herein would not have been possible.

\section{REFERENCES}

Bakos, G., Noyes, R. W., Kovacs, G., et al. 2004, PASP, 116, 266 Barbieri, M., Alonso, R., Laughlin, G., et al. 2007, A\&A, 476, L13 Barge, P., Baglin, A., Auvergne, M., et al. 2008, A\&A, 482, L17 Bessell, M. S. 2000, PASP, 112, 961

Bodenheimer, P., Laughlin, G., \& Lin, D. N. C. 2003, ApJ, 592, 555

Borucki, W. J., Koch, D. G., Basri, G., et al. 2011, ApJ, 728, 117

Bouchy, F., Udry, S., Mayor, M., et al. 2005, A\&A, 444, L15

Butler, R. P., Marcy, G. W., Williams, E., et al. 1996, PASP, 108, 500

Castellano, T., Jenkins, J., Trilling, D. E., Doyle, L., \& Koch, D. 2000, ApJ, 532, L51

Charbonneau, D., Brown, T. M., Latham, D. W., \& Mayor, M. 2000, ApJ, 529, L45
Colon, K. D., Ford, E. B., Redfield, S., et al. 2010, MNRAS, submitted (arXiv:1008.4800)

Dawson, R. I., \& Fabrycky, D. C. 2010, ApJ, 722, 937

Demarque, P., Woo, J., Kim, Y., \& Yi, S. K. 2004, ApJS, 155, 667

Everett, M. E., \& Howell, S. B. 2001, PASP, 113, 1428

Fossey, S. J., Waldmann, I. P., \& Kipping, D. M. 2009, MNRAS, 396, L16

Hébrard, G., \& Lecavelier Des Etangs, A. 2006, A\&A, 445, 341

Henry, G. W. 1999, PASP, 111, 845

Henry, G. W., Howard, A. W., Marcy, G. W., Fischer, D. A., \& Johnson, J. A. 2011, ApJ, submitted (arXiv:1109.2549)

Henry, G. W., Marcy, G. W., Butler, R. P., \& Vogt, S. S. 2000, ApJ, 529, L41

Howard, A. W., Johnson, J. A., Marcy, G. W., et al. 2009, ApJ, 696, 75

Howard, A. W., Johnson, J. A., Marcy, G. W., et al. 2010, ApJ, 721, 1467

Isaacson, H., \& Fischer, D. A. 2010, ApJ, 725, 875

Kane, S. R., Howard, A. W., Pilyavsky, G., et al. 2011, ApJ, 733, 1

Kane, S. R., Mahadevan, S., von Braun, K., Laughlin, G., \& Ciardi, D. R. 2009, PASP, 121, 1386

Kane, S. R., \& von Braun, K. 2008, ApJ, 689, 492

Marcy, G. W., \& Butler, R. P. 1992, PASP, 104, 270

Marcy, G. W., Butler, R. P., Vogt, S. S., Fischer, D., \& Liu, M. C. 1999, ApJ, 520,239

Marcy, G. W., Butler, R. P., Vogt, S. S., et al. 2001, ApJ, 555, 418

Marcy, G. W., Butler, R. P., Vogt, S. S., et al. 2005, ApJ, 619, 570

McCullough, P. R., Stys, J. E., Valenti, J. A., et al. 2005, PASP, 117, 783

Moutou, C., Hébrard, G., Bouchy, F., et al. 2009, A\&A, 498, L5

Pollacco, D. L., Skillen, I., Collier Cameron, A., et al. 2006, PASP, 118, 1407

Queloz, D., Henry, G. W., Sivan, J. P., et al. 2001, A\&A, 379, 279

Redfield, S., Endl, M., Cochran, W. D., \& Koesterke, L. 2008, ApJ, 673, L87

Reffert, S., \& Quirrenbach, A. 2006, A\&A, 449, 699

Reffert, S., \& Quirrenbach, A. 2011, A\&A, 527, A140

Robichon, N., \& Arenou, F. 2000, A\&A, 355, 295

Sahlmann, J., Ségransan, D., Queloz, D., et al. 2011, A\&A, 525, A95

Sato, B., Fischer, D. A., Henry, G. W., et al. 2005, ApJ, 633, 465

Sing, D. K., Désert, J.-M., Fortney, J. J., et al. 2011, A\&A, 527, A73

Snellen, I. A. G., Albrecht, S., de Mooij, E. J. W., \& Le Poole, R. S. 2008, A\&A, 487,357

Stetson, P. B. 1987, PASP, 99, 191

Udry, S., Mayor, M., Naef, D., et al. 2002, A\&A, 390, 267

Valenti, J. A., Butler, R. P., \& Marcy, G. W. 1995, PASP, 107, 966

Valenti, J. A., \& Fischer, D. A. 2005, ApJS, 159, 141

Valenti, J. A., Fischer, D., Marcy, G. W., et al. 2009, ApJ, 702, 989

Valenti, J. A., \& Piskunov, N. 1996, A\&AS, 118, 595

van Belle, G. T., \& von Braun, K. 2009, ApJ, 694, 1085

van Leeuwen, F. 2007, A\&A, 474, 653

Veras, D., \& Armitage, P. J. 2007, ApJ, 661, 1311

Veras, D., \& Ford, E. B. 2010, ApJ, 715, 803

Vidal-Madjar, A., Sing, D. K., Lecavelier Des Etangs, A., et al. 2011, A\&A, 527, A110

Vogt, S. S., Allen, S. L., Bigelow, B. C., et al. 1994, Proc. SPIE, 2198, 362

von Braun, K., Kane, S. R., \& Ciardi, D. R. 2009, ApJ, 702, 779

Winn, J. N., Matthews, J. M., Dawson, R. I., et al. 2011, ApJ, 737, L18

Wright, J. T. 2005, PASP, 117, 657

Wright, J. T., \& Howard, A. W. 2009, ApJS, 182, 205

Wright, J. T., Upadhyay, S., Marcy, G. W., et al. 2009, ApJ, 693, 1084 